\title{
Soft Fuzzy Incline
}

\author{
Yanwei Shi \\ School of Intelligent Science and Information Engineering \\ Xi'an Peihua University \\ Xi'an, China
}

\begin{abstract}
The main purpose of this paper is to study the theory of soft fuzzy incline. At first, as generalization of soft sets initiated by Molodtsov, the concepts of soft fuzzy set and its extension are defined. Then three kinds of operations, complement, intersection and union, with soft fuzzy sets are studied. Finally, the definition of soft fuzzy incline is proposed and sufficient and necessary conditions of which soft fuzzy set is soft fuzzy incline are shown.
\end{abstract} incline

Keywords—soft fuzzy set; extension; soft fuzzy equal; soft fuzzy

\section{INTRODUCTION}

A number of practical problems within economics, engineering, etc. involve data that contain uncertainties. Nowadays, many theories, such as probability theory, fuzzy set theory[1], theory of interval mathematics[2], vague set theory[3], rough set theory[4], etc. have been proposed for dealing with uncertainties. However, these theories are associated with inherent limitations, as pointed out in [5]. Molodtsov[5] initiated the concept of soft set theory as a new mathematical tool for dealing with uncertainties which is free from the above limitations. The soft set theory has a rich potential for applications and has been applied in several directions.

Soft sets can approach to the real life in one way. However, there are also difficulties in soft set theory. For example, the value-set [6] of a soft set is still a family of sets. It can't describe the degree in the fact, since the membership of elements in a set is assessed in binary terms according to a bivalent condition - an element either belongs or does not belong to the set. By contrast, fuzzy set theory permits the gradual assessment of the membership of elements in a set. This is described with the aid of a membership function valued in the real unit interval $[0,1]$. Fuzzy sets generalized classical sets, since the indicator functions of classical sets are special cases of the membership functions of fuzzy sets, if the latter only take value 0 or 1 .

Consequently, in the present paper, we generalize soft sets to the soft fuzzy sets. To put two soft fuzzy sets to a common parameters set, their extensions are defined in a natural way. Using their extensions, some operations with them are introduced and their basic properties are investigated. At last, the definition of soft fuzzy incline is proposed and sufficient and necessary conditions of which soft fuzzy set is soft fuzzy incline are shown.

\author{
Chunhui Ma \\ School of Science \\ Xi'an University of Architecture and Technology \\ Xi'an, China
}

Although soft fuzzy sets had been mentioned and applied to decision making problems in [7], only few relations and operations about them were given and more detail about two soft fuzzy sets over different supported sets didn't be discussed. However, using the extension provided in the present paper, more properties can be easily obtained and our work in this paper is completely theoretical.

\section{SOFT FUZZY SET AND OPERATIONS}

In the reference [5], soft set was defined as follows. Let $U$ be an initial universe set and $E$ be a set of parameters. And $P(U)$ denotes the power set of $U$ (i.e. $P(U)$ is the family of all subsets of the set $U$ ) and $A \subseteq E$.

Definition 2.1 A pair $(F, A)$ is called a soft set over $U$ if and only if $F$ is a mapping given by $F: A \rightarrow P(U)$.

In other words, a soft set over $U$ is a parameterized family of subsets of the universe set $U$.

To describe the degree, a new concept needs to give.

Let $U$ be an initial universe set and $E$ be a set of parameters. And $F(U)$ denotes the set of all fuzzy subsets on $U$ (i.e. $F(U)$ is the family of all mapping from the set $U$ to the unit interval $[0,1])$ and $A \subseteq E$.

Definition 2.2 A pair $(\widetilde{F}, A)$ is called a soft fuzzy set over $U$ if and only if $\widetilde{F}$ is a mapping given by $\widetilde{F}: A \rightarrow F(U)$.

In other words, a soft fuzzy set over $U$ is a parameterized family of fuzzy subsets on the universe set $U$.

Some basic operations with soft fuzzy sets will be discussed.

Definition 2.3 Two soft fuzzy sets $(\widetilde{F}, A)$ and $(\widetilde{G}, B)$ over a common universe set $U$ are said to be equal if $A=B$ and $\widetilde{F}(e)=\widetilde{G}(e)$ for each $e \in A$ (or $B$ ).

Clearly, if two soft fuzzy sets are equal, they need same parameters set. For different parameters sets, the following definition can be used. 
Definition 2.4 Soft fuzzy set $\left(\widetilde{F}_{D}^{*}, D\right)$, where $D \subseteq E$, is said to be the extension of a soft fuzzy set $(\widetilde{F}, A)$ over a common universe set $U$, if and only if $A \subseteq D$ and for every $e \in D$

$$
\widetilde{F}_{D}^{*}(e)=\left\{\begin{array}{c}
\widetilde{F}(e), \quad \text { if } e \in A \\
M, \quad \text { if } e \in D \backslash A
\end{array}\right.
$$

where $M \in F(U)$ and $M(u)=0.5$ for each $u \in U$.

In this case, the extension $\left(\widetilde{F}_{D}^{*}, D\right)$ of soft fuzzy set $(\widetilde{F}, A)$ can be denoted by $\operatorname{EXT}_{D}(\widetilde{F}, A)$. Particularly, the index is omitted if $D=E$, that is,

$$
\operatorname{EXT}(\widetilde{F}, A)=\operatorname{EXT}_{E}(\widetilde{F}, A)=\left(\widetilde{F}_{E}^{*}, E\right)
$$

Using the extension of soft fuzzy set, some soft fuzzy sets with different parameters can be extended to a same parameters set to operate.

Definition 2.5 Two soft fuzzy sets $(\widetilde{F}, A)$ and $(\widetilde{G}, B)$ over a common universe set $U$ are said to be soft fuzzy equal denoted by $(\widetilde{F}, A) \stackrel{\sim}{=}(\widetilde{G}, B)$, if and only if there exists $D \subseteq E$ such that $\operatorname{EXT}_{D}(\widetilde{F}, A)=\operatorname{EXT}_{D}(\widetilde{G}, B)$.

It is obvious that soft fuzzy equal relation is an equivalent relation on the family of all soft fuzzy sets over a common universe set $U$.

Theorem 2.6 Let $(\widetilde{F}, A)$ be a soft fuzzy set over universe set $U$.

(1) $\operatorname{EXT}_{A}(\widetilde{F}, A)=(\widetilde{F}, A)$.

(2) $\operatorname{EXT}_{D}(\widetilde{F}, A) \stackrel{\sim}{=}(\widetilde{F}, A)$ for all $A \subseteq D$.

(3) $\left.\operatorname{EXT}_{D_{2}}\left(\operatorname{EXT}_{D_{1}}(\widetilde{F}, A)\right) \stackrel{\tilde{=}}{=} \widetilde{F}, A\right)$ for all

$$
A \subseteq D_{1} \subseteq D_{2} \text {. }
$$

(4) $\operatorname{EXT}_{D_{2}}\left[\operatorname{EXT}_{D_{1}}(\widetilde{F}, A)\right]=\operatorname{EXT}_{D_{1}}\left[\operatorname{EXT}_{D_{2}}(\widetilde{F}, A)\right]$, if and only if $D_{1}=D_{2}$.

Proof. (1) $\operatorname{EXT}_{A}(\widetilde{F}, A)=\left(\widetilde{F}_{A}^{*}, A\right)$. By the definition of $\widetilde{F}_{A}^{*}, \widetilde{F}_{A}^{*}(e)=\widetilde{F}(e)$ for each $e \in A$, i.e. $\widetilde{F}_{A}^{*}=\widetilde{F}$. Thus, $\left(\widetilde{F}_{A}^{*}, A\right)=(\widetilde{F}, A)$, that is, $\operatorname{EXT}_{A}(\widetilde{F}, A)=(\widetilde{F}, A)$.

(2) $\operatorname{EXT}_{D}(\widetilde{F}, A)$ is well defined, since $D \supseteq A$. And for every $\quad D \supseteq A \quad, \quad \operatorname{EXT}_{D}(\widetilde{F}, A)=\left(\widetilde{F}_{D}^{*}, D\right) \quad$ and
$\operatorname{EXT}_{D}\left[\operatorname{EXT}_{D}(\widetilde{F}, A)\right]=\operatorname{EXT}_{D}\left(\widetilde{F}_{D}^{*}, D\right)=\left(\widetilde{F}_{D}^{*}, D\right) \quad$ by (1). So, $\operatorname{EXT}_{D}\left[\operatorname{EXT}_{D}(\widetilde{F}, A)\right]=\operatorname{EXT}_{D}(\widetilde{F}, A)$, that is, $\operatorname{EXT}_{D}(\widetilde{F}, A) \stackrel{\sim}{=}(\widetilde{F}, A)$ for all $A \subseteq D$.

(3) It is well defined for $A \subseteq D_{1} \subseteq D_{2}$. By (2), $\operatorname{EXT}_{D_{1}}(\widetilde{F}, A) \stackrel{\sim}{=}(\widetilde{F}, A)$, and

$$
\begin{array}{r}
\operatorname{EXT}_{D_{2}}\left(\operatorname{EXT}_{D_{1}}(\widetilde{F}, A)\right)=\operatorname{EXT}_{D_{2}}\left(\widetilde{F}_{D_{1}}^{*}, D_{1}\right) \\
\stackrel{\sim}{=}\left(\widetilde{F}_{D_{1}}^{*}, D_{1}\right)=\operatorname{EXT}_{D_{1}}(\widetilde{F}, A),
\end{array}
$$

So $\operatorname{EXT}_{D_{2}}\left(\operatorname{EXT}_{D_{1}}(\widetilde{F}, A)\right) \stackrel{\sim}{=}(\widetilde{F}, A)$.

(4) By (3),

$\operatorname{EXT}_{D_{2}}\left[\operatorname{EXT}_{D_{1}}(\widetilde{F}, A)\right]=\operatorname{EXT}_{D_{1}}\left[\operatorname{EXT}_{D_{2}}(\widetilde{F}, A)\right]$,

if and only if $\left(\widetilde{F}_{D_{2}}^{*}, D_{2}\right)=\left(\widetilde{F}_{D_{1}}^{*}, D_{1}\right)$, if and only if $D_{1}=D_{2}$.

From Definition 2.3 and Definition 2.5, it should be found that the equal relation $=$ and soft fuzzy equal relation $\stackrel{\sim}{=}$ are different. But it can be implied that two soft fuzzy sets are soft fuzzy equal, if they are equal.

Theorem 2.7 Let $(\widetilde{F}, A)$ and $(\widetilde{G}, B)$ be two soft fuzzy sets over a common universe set $U$. If $(\widetilde{F}, A) \stackrel{\sim}{=}(\widetilde{G}, B)$, then

(1) $\widetilde{F}(e)=\widetilde{G}(e)$ for all $e \in A \cap B$.

(2) $\widetilde{F}(e)=M$ for all $e \in A \backslash B$.

(3) $\widetilde{G}(e)=M$ for all $e \in B \backslash A$.

Proof. Since $(\widetilde{F}, A) \tilde{=}(\widetilde{G}, B)$, then there exists $D \subseteq E$ such that $\operatorname{EXT}_{D}(\widetilde{F}, A)=\operatorname{EXT}_{D}(\widetilde{G}, B)$. Thus, $A, B \subseteq D$ and $\widetilde{F}_{D}^{*}(e)=\widetilde{G}_{D}^{*}(e)$ for all $e \in D$.

(1) By the definition of extension, for each $e \in A \cap B$, $\widetilde{F}(e)=\widetilde{G}(e)$.

(2) For every $e \in A \backslash B$,

(3) Similar to (2).

$$
\widetilde{F}(e)=\widetilde{F}_{D}^{*}(e)=\widetilde{G}_{D}^{*}(e)=M .
$$

Corollary 2.8 Let $(\widetilde{F}, A)$ and $(\widetilde{G}, B)$ be two soft fuzzy sets over a common universe set $U$. If $(\widetilde{F}, A) \stackrel{\sim}{=}(\widetilde{G}, B)$, then

(1) $(\widetilde{F}, A)=(\widetilde{G}, B)$ if and only if $A=B$. 
(2) if $A \cap B=\varnothing, \widetilde{F}\left(e_{A}\right)=\widetilde{G}\left(e_{B}\right)=M$ for each $e_{A} \in A$ and $e_{B} \in B$.

(3) $\bigcap\left\{D \subseteq E \mid \operatorname{EXT}_{D}(\widetilde{F}, A)\right.$

$$
\left.=\operatorname{EXT}_{D}(\widetilde{G}, B)\right\}=A \cup B \text {. }
$$

Proof. (1) If $(\widetilde{F}, A)=(\widetilde{G}, B)$, by the definition of equal, $A=B$. Conversely, let $A=B$. Then for every $e \in A$ (or $B$ ), $\widetilde{F}(e)=\widetilde{G}(e)$ by Theorem 2.7(1). Thus, $\widetilde{F}=\widetilde{G}$. So $(\widetilde{F}, A)=(\widetilde{G}, B)$.

(2) It can be easily gotten by Theorem 2.7(2) and (3).

(3) For every $D \subseteq E$ such that

$\operatorname{EXT}_{D}(\widetilde{F}, A)=\operatorname{EXT}_{D}(\widetilde{G}, B)$, by the definition of extension, $D \supseteq A$ and $D \supseteq B$. Then $D \supseteq A \cup B$. Thus, $\bigcap\left\{D \subseteq E \mid \operatorname{EXT}_{D}(\widetilde{F}, A)=\operatorname{EXT}_{D}(\widetilde{G}, B)\right\} \supseteq A \cup B$.

On the other hand, for $\operatorname{EXT}_{A \cup B}(\widetilde{F}, A)$ and $\operatorname{EXT}_{A \cup B}(\widetilde{G}, B)$,

$$
\widetilde{F}_{A \cup B}^{*}=\left\{\begin{array}{c}
\widetilde{F}(e), \quad \text { if } e \in A \\
M, \quad \text { if } e \in B \backslash A
\end{array}\right.
$$

and

$$
\widetilde{G}_{A \cup B}^{*}=\left\{\begin{array}{c}
\widetilde{G}(e), \quad \text { if } e \in B \\
M, \quad \text { if } e \in A \backslash B
\end{array} .\right.
$$

By Theorem 2.7, $\widetilde{F}_{A \cup B}^{*}=\widetilde{G}_{A \cup B}^{*}$. Then

$$
\left(\widetilde{F}_{A \cup B}^{*}, A \cup B\right)=\left(\widetilde{G}_{A \cup B}^{*}, A \cup B\right),
$$

that is, $\operatorname{EXT}_{A \cup B}(\widetilde{F}, A)=\operatorname{EXT}_{A \cup B}(\widetilde{G}, B)$.

So,

$\bigcap\left\{D \subseteq E \mid \operatorname{EXT}_{D}(\widetilde{F}, A)=\operatorname{EXT}_{D}(\widetilde{G}, B)\right\} \subseteq A \cup B \quad$

Therefore,

$\bigcap\left\{D \subseteq E \mid \operatorname{EXT}_{D}(\widetilde{F}, A)=\operatorname{EXT}_{D}(\widetilde{G}, B)\right\}=A \cup B$.

From Theorem 2.6 and Corollary 2.8, the following theorem is straightforward.

Theorem 2.9 Two soft fuzzy sets $(\widetilde{F}, A)$ and $(\widetilde{G}, B)$ over a common universe set $U$ are soft fuzzy equal, if and only if $\widetilde{F}_{A \cup B}^{*}=\widetilde{G}_{A \cup B}^{*}$.

Next, three kind of operations - complement, intersection and union - with soft fuzzy sets are studied.
Definition 2.10 Let $(\widetilde{F}, A)$ and $(\widetilde{G}, B)$ be two soft fuzzy sets over a common universe set $U$.

(1) The complement of $(\widetilde{F}, A)$ is denoted by $(\widetilde{F}, A)^{c}$ and is defined by $(\widetilde{F}, A)^{c}=\left(\widetilde{F}^{c}, A\right)$ such that $\widetilde{F}^{c}: A \rightarrow F(U)$ is a mapping given by $\widetilde{F}^{c}(e)=1_{U}-\widetilde{F}(e)$ for all $e \in A$ and $1_{U} \in F(U)$ such that $1_{U}(u)=1$ for each $u \in U$

(2) The intersection of $(\widetilde{F}, A)$ and $(\widetilde{G}, B)$ is denoted by $(\widetilde{F}, A) \sim(\widetilde{G}, B)$ and is defined by

$$
(\widetilde{F}, A) \sim(\widetilde{G}, B)=(\widetilde{H}, A \cup B)
$$

such that $\widetilde{H}(e)=\widetilde{F}_{A \cup B}^{*}(e) \wedge \widetilde{G}_{A \cup B}^{*}(e)$ for all $e \in A \cup B$, where $\widetilde{F}_{A \cup B}^{*}$ and $\widetilde{G}_{A \cup B}^{*}$ are the extensions of $\widetilde{F}$ and $\widetilde{G}$ to $A \cup B$, respectively, and $\wedge$ is the intersection operation on $F(U)$.

(3) The union of $(\widetilde{F}, A)$ and $(\widetilde{G}, B)$ is denoted by $(\widetilde{F}, A) \widetilde{\vee}(\widetilde{G}, B)$ and is defined by

$$
(\widetilde{F}, A) \widetilde{\vee}(\widetilde{G}, B)=(\widetilde{H}, A \cup B)
$$

such that $\widetilde{H}(e)=\widetilde{F}_{A \cup B}^{*}(e) \vee \widetilde{G}_{A \cup B}^{*}(e)$ for every $e \in A \cup B$, where $\widetilde{F}_{A \cup B}^{*}$ and $\widetilde{G}_{A \cup B}^{*}$ are the extensions of $\widetilde{F}$ and $\widetilde{G}$ to $A \cup B$, respectively, and $\vee$ is the union operation on $F(U)$.

Some properties of these operations will be discussed. Here, suppose $D \subseteq E$ such that $\mathrm{EXT}_{D}$ is well defined. It will not be mentioned again in the following.

Lemma 2.11 Let $(\widetilde{F}, A)$ and $(\widetilde{G}, B)$ be soft fuzzy sets over the universe set $U$.

(1) $\operatorname{EXT}_{D}\left[(\widetilde{F}, A)^{c}\right]=\left[\operatorname{EXT}_{D}(\widetilde{F}, A)\right]^{c}$.

(2) $\operatorname{EXT}_{D}[(\widetilde{F}, A) \widetilde{\wedge}(\widetilde{G}, B)]$

$$
=\operatorname{EXT}_{D}(\widetilde{F}, A) \tilde{\wedge} \operatorname{EXT}_{D}(\widetilde{G}, B) .
$$

(3) $\operatorname{EXT}_{D}[(\widetilde{F}, A) \widetilde{\vee}(\widetilde{G}, B)]$

$$
=\operatorname{EXT}_{D}(\widetilde{F}, A) \tilde{\vee} \operatorname{EXT}_{D}(\widetilde{G}, B) .
$$
2.8(1).

Proof. Straightforward by Theorem 2.6(2) and Corollary

Corollary 2.12 Let $(\widetilde{F}, A)$ and $(\widetilde{G}, B)$ be soft fuzzy sets over the universe set $U$. 
(1) $\operatorname{EXT}_{A \cup B}\left[(\widetilde{F}, A)^{c}\right]=(\widetilde{F}, A)^{c}$.

(2) $\operatorname{EXT}_{A \cup B}[(\widetilde{F}, A) \wedge(\widetilde{G}, B)]=(\widetilde{F}, A) \tilde{\wedge}(\widetilde{G}, B)$.

(3) $\operatorname{EXT}_{A \cup B}[(\widetilde{F}, A) \widetilde{\vee}(\widetilde{G}, B)]=(\widetilde{F}, A) \widetilde{\vee}(\widetilde{G}, B)$.

Proof. It is straightforward by Theorem 2.6(1) and Lemma 2.11 above.

The most basic properties of these operators will be given in the following theorem and later notes.

Theorem 2.13 Let $(\widetilde{F}, A),(\widetilde{G}, B)$ and $(\widetilde{H}, C)$ be soft fuzzy sets over the universe set $U$.

(1) $\left[(\widetilde{F}, A)^{c}\right]^{c}=(\widetilde{F}, A)$.

(2) $(\widetilde{F}, A) \sim(\widetilde{G}, B)=(\widetilde{G}, B) \sim(\widetilde{F}, A)$.

(3) $[(\widetilde{F}, A) \tilde{\wedge}(\widetilde{G}, B)] \tilde{\wedge}(\widetilde{H}, C)$

$$
=(\widetilde{F}, A) \sim[(\widetilde{G}, B) \sim(\widetilde{H}, C)] .
$$

(4) $(\widetilde{F}, A) \widetilde{\wedge}[(\widetilde{G}, B) \widetilde{\vee}(\widetilde{H}, C)]$

$$
=[(\widetilde{F}, A) \tilde{\wedge}(\widetilde{G}, B)] \tilde{\vee}[(\widetilde{F}, A) \tilde{\wedge}(\widetilde{H}, C)] .
$$

(5) $[(\widetilde{F}, A) \widetilde{\wedge}(\widetilde{G}, B)]^{c}=(\widetilde{F}, A)^{c} \widetilde{\vee}(\widetilde{G}, B)^{c}$.

Proof. (1) By Definition 2.10(1),

$$
\left[(\widetilde{F}, A)^{c}\right]^{c}=\left(\widetilde{F}^{c}, A\right)^{c}=\left(\left(\widetilde{F}^{c}\right)^{c}, A\right)
$$

and $\quad\left(\widetilde{F}^{c}\right)^{c}(e)=1_{U}-\widetilde{F}^{c}(e)=1_{U}-\left(1_{U}-\widetilde{F}(e)\right)=\widetilde{F}(e)$ for each $e \in A$. So, $\left[(\widetilde{F}, A)^{c}\right]^{c}=(\widetilde{F}, A)$.

(2) Suppose $(\widetilde{F}, A) \sim(\widetilde{G}, B)=(\widetilde{K}, A \cup B) \quad$ and $(\widetilde{G}, B) \sim(\widetilde{F}, A)=(\widetilde{L}, A \cup B)$. By Definition 2.10(2), for all $e \in A \cup B$,

$$
\begin{aligned}
& \widetilde{K}(e)=\widetilde{F}_{A \cup B}^{*}(e) \wedge \widetilde{G}_{A \cup B}^{*}(e) \\
& =\widetilde{G}_{A \cup B}^{*}(e) \wedge \widetilde{F}_{A \cup B}^{*}(e)=\widetilde{L}(e) .
\end{aligned}
$$

That is, $\widetilde{K}=\widetilde{L}$. So, $(\widetilde{F}, A) \sim(\widetilde{G}, B)=(\widetilde{G}, B) \sim(\widetilde{F}, A)$.

(3) Suppose $(\widetilde{F}, A) \widetilde{\wedge}(\widetilde{G}, B)=(\widetilde{K}, A \cup B) \quad$ and $(\widetilde{K}, A \cup B) \widetilde{\wedge}(\widetilde{H}, C)=(\widetilde{L}, A \cup B \cup C)$. By Definition 2.6(2), for all $e \in A \cup B \cup C$,

$$
\begin{aligned}
& \tilde{L}(e)=\widetilde{K}_{A \cup B \cup C}^{*}(e) \wedge \widetilde{H}_{A \cup B \cup C}^{*}(e) \\
& =\widetilde{F}_{A \cup B \cup C}^{*}(e) \wedge \widetilde{G}_{A \cup B \cup C}^{*}(e) \wedge \widetilde{H}_{A \cup B \cup C}^{*}(e)
\end{aligned}
$$

by Corollary 2.12(2). Similarly, suppose
$(\widetilde{F}, A) \tilde{\wedge}[(\widetilde{G}, B) \widetilde{\wedge}(\widetilde{H}, C)]=\left(\widetilde{L^{\prime}}, A \cup B \cup C\right)$.

Also, for every $e \in A \cup B \cup C$,

$$
{\widetilde{L^{\prime}}}^{\prime}(e)=\widetilde{F}_{A \cup B \cup C}^{*}(e) \wedge \widetilde{G}_{A \cup B \cup C}^{*}(e) \wedge \widetilde{H}_{A \cup B \cup C}^{*}(e) .
$$

So, $[(\widetilde{F}, A) \sim(\widetilde{G}, B)] \widetilde{\wedge}(\widetilde{H}, C)$

$$
=(\widetilde{F}, A) \widetilde{\wedge}[(\widetilde{G}, B) \widetilde{\wedge}(\widetilde{H}, C)] .
$$

(4) Suppose $(\widetilde{G}, B) \widetilde{\vee}(\widetilde{H}, C)=(\widetilde{K}, B \cup C)$ and $(\widetilde{F}, A) \sim(\widetilde{K}, B \cup C)=(\widetilde{L}, A \cup B \cup C)$.

By Corollary 2.8(2) and (3),

$$
\begin{gathered}
\tilde{L}(e)=\widetilde{F}_{A \cup B \cup C}^{*}(e) \wedge \widetilde{K}_{A \cup B \cup C}^{*}(e) \\
=\widetilde{F}_{A \cup B \cup C}^{*}(e) \wedge\left[\widetilde{G}_{A \cup B \cup C}^{*}(e) \vee \widetilde{H}_{A \cup B \cup C}^{*}(e)\right] .
\end{gathered}
$$

On the other hand, suppose

$$
\begin{aligned}
& (\widetilde{F}, A) \sim(\widetilde{\wedge}, B)=\left(\widetilde{K^{\prime}}, A \cup B\right), \\
& (\widetilde{F}, A) \sim(\widetilde{H}, C)=\left(\widetilde{K^{\prime \prime}}, A \cup C\right)
\end{aligned}
$$

and

$$
\left(\widetilde{K^{\prime}}, A \cup B\right) \widetilde{\vee}\left(\widetilde{K^{\prime \prime}}, A \cup C\right)=\left(\widetilde{L^{\prime}}, A \cup B \cup C\right) .
$$

For each $e \in A \cup B \cup C$,

$\widetilde{L^{\prime}}(e)=\widetilde{K}^{\prime}{ }^{*} \cup B \cup C(e) \vee{\widetilde{K^{\prime \prime}}}_{A \cup B \cup C}^{*}(e)$

$=\left[\widetilde{F}_{A \cup B \cup C}^{*}(e) \wedge \widetilde{G}_{A \cup B \cup C}^{*}(e)\right] \vee\left[\widetilde{F}_{A \cup B \cup C}^{*}(e) \wedge \widetilde{H}_{A \cup B \cup C}^{*}(e)\right]$

$=\widetilde{F}_{A \cup B \cup C}^{*}(e) \wedge\left[\widetilde{G}_{A \cup B \cup C}^{*}(e) \vee \widetilde{H}_{A \cup B \cup C}^{*}(e)\right]$.

So, $(\widetilde{F}, A) \widetilde{\wedge}[(\widetilde{G}, B) \widetilde{\vee}(\widetilde{H}, C)]$

$$
=[(\widetilde{F}, A) \widetilde{\wedge}(\widetilde{G}, B)] \widetilde{\vee}[(\widetilde{F}, A) \widetilde{\wedge}(\widetilde{H}, C)] .
$$

(5) Suppose $(\widetilde{F}, A) \widetilde{\wedge}(\widetilde{G}, B)=(\widetilde{K}, A \cup B) \quad$ and $(\widetilde{F}, A)^{c} \widetilde{\sim}(\widetilde{G}, B)^{c}=(\widetilde{L}, A \cup B)$. By Definition 2.10 and Corollary 2.12, for every $e \in A \cup B$,

$$
\begin{aligned}
& \widetilde{K}^{c}(e)=1_{U}-\widetilde{K}(e)=1_{U}-\left[\widetilde{F}_{A \cup B}^{*}(e) \wedge \widetilde{G}_{A \cup B}^{*}(e)\right] \\
& =\left[1_{U}-\widetilde{F}_{A \cup B}^{*}(e)\right] \vee\left[1_{U}-\widetilde{G}_{A \cup B}^{*}(e)\right] \\
& =\left(\widetilde{F}^{*}\right)_{A \cup B}^{c}(e) \vee\left(\widetilde{G}^{*}\right)_{A \cup B}^{c}(e) \\
& =\left(\widetilde{F}^{c}\right)_{A \cup B}^{*}(e) \vee\left(\widetilde{G}^{c}\right)_{A \cup B}^{*}(e)=L(e) .
\end{aligned}
$$

Thus, $[(\widetilde{F}, A) \widetilde{\wedge}(\widetilde{G}, B)]^{c}=(\widetilde{F}, A)^{c} \sim \widetilde{\vee}(\widetilde{G}, B)^{c}$. 


\section{SOFT FUZZY INCLINE}

In the last part of this paper, soft fuzzy incline, will be discussed.

Incline is a generalization of Boolean and fuzzy algebras (see [2]). It is a special type of semi-ring.

Definition 3.1 Let $E$ be a nonempty set with two binary operations denoted by + and $*$. If the following conditions are satisfied for all $x, y, z \in E$ :

(1) $x+y=y+x, x^{*} y=y^{*} x$

(2) $x+(y+z)=(x+y)+z$, $x^{*}\left(y^{*} z\right)=\left(x^{*} y\right) * z$

(3) $x^{*}(y+z)=\left(x^{*} y\right)+\left(x^{*} z\right)$;

(4) $x+x=x$;

(5) $x+\left(x^{*} y\right)=x$,

$(E,+, *)$ is called a incline (algebra) on $E$.

If a subset $S$ of an incline $(E,+, *)$ is closed under + and $*,(S,+, *)$ is called a subincline of the incline $(E,+, *)$.

In references [4,7], the fuzzy algebra structures are discussed. And, the following concept, fuzzy subincline, was provided in [7].

Definition 3.2 Let $\left(E,+{ }^{*}\right)$ be an incline and $F(E)$ denote the set of all fuzzy subsets on $E . S \in F(E)$ is called a fuzzy subincline of the incline $(E,+, *)$ if the nonempty level subset $S_{t}=\{x \in E \mid S(x) \geq t\} \quad(\forall t \in[0,1])$ of $S$ is a subincline of $E$.

And a characterization of fuzzy subincline was also provided in [7].

Theorem 3.3 $S \in F(E)$ is a fuzzy subincline of the incline $(E,+, *)$ if and only if, for all $x, y \in E$, $S(x) \wedge S(y) \leq S(x+y) \wedge S\left(x^{*} y\right)$.

Here, a simple example which we will used is given.

Example 3.4 $M$ is a fuzzy subincline of the incline $\left(E,+,^{*}\right)$, where $M \in F(E)$ and $M(e)=0.5$ for all $e \in E$.

To define soft fuzzy incline, suppose that the universe set and the parameters set are both a given incline $\left(E,+,^{*}\right)$.

Definition 3.5 A soft fuzzy set $(\widetilde{F}, A)$ over an incline $\left(E,+,^{*}\right)$, where $A \subseteq E$, is called a soft fuzzy incline if $\widetilde{F}(e)$ is a fuzzy subincline of $E$ for each $e \in A$.
Since not every fuzzy subset of the incline $\left(E,+,{ }^{*}\right)$ is a fuzzy subincline, then not all soft fuzzy sets over $E$ are soft fuzzy incline. The counterexamples are omitted for it is so clear.

Theorem 3.6 Let $\left(E,+{ }^{*}\right)$ be an incline. The followings are equivalent:

(1) $(\widetilde{F}, A)$ is a soft fuzzy incline over $E$.

(2) $\operatorname{EXT}_{D}(\widetilde{F}, A)$ is a soft fuzzy incline over $E$ for every $D \supseteq A$.

(3) There exits $D \supseteq A$ such that $\operatorname{EXT}_{D}(\widetilde{F}, A)$ is a soft fuzzy incline over $E$.

Proof. $(1) \Rightarrow(2)$ Let $(\widetilde{F}, A)$ be a soft fuzzy incline over $E$. For every $D \supseteq A, \operatorname{EXT}_{D}(\widetilde{F}, A)=\left(\widetilde{F}_{D}^{*}, D\right)$, where

$$
\widetilde{F}_{D}^{*}(e)=\left\{\begin{array}{c}
\widetilde{F}(e), \quad \text { if } e \in A \\
M, \quad \text { if } e \in D \backslash A
\end{array}\right.
$$

Sine $(\widetilde{F}, A)$ is a soft fuzzy incline, $\widetilde{F}(e)$ is a fuzzy subincline for each $e \in A$. And $M$ is a fuzzy subincline (see Example 3.4). Thus, for all $e \in D, \widetilde{F}_{D}^{*}(e)$ is a fuzzy subincline. That is, $\operatorname{EXT}_{D}(\widetilde{F}, A)$ is a soft fuzzy Incline over $E$.

(2) $\Rightarrow$ (3) Straightforward.

(3) $\Rightarrow(1)$ Let $\operatorname{EXT}_{D}(\widetilde{F}, A)$ be a soft fuzzy incline over $E$. That is, for every $e \in D, \widetilde{F}_{D}^{*}(e)$ is a fuzzy subincline. Then for all $e \in A, \widetilde{F}_{D}^{*}(e)=\widetilde{F}(e)$ is also a fuzzy subincline. So, $(\widetilde{F}, A)$ is a soft fuzzy incline over $E$.

Corollary 3.7 If $(\widetilde{F}, A)$ is a soft fuzzy incline, and $(\widetilde{F}, A) \stackrel{\sim}{=}(\widetilde{G}, B)$, then $(\widetilde{G}, B)$ is also a soft fuzzy incline.

Proof. Let $(\widetilde{F}, A)$ be a soft fuzzy incline over $E$ and $(\widetilde{F}, A) \stackrel{\sim}{=}(\widetilde{G}, B)$. By Definition 3.5 , there exists $D \subseteq E$ such that $\operatorname{EXT}_{D}(\widetilde{F}, A)=\operatorname{EXT}_{D}(\widetilde{G}, B)$. Therefore, $\operatorname{EXT}_{D}(\widetilde{F}, A)$ is a soft fuzzy incline over $E$ by Theorem 2.13. So, $(\widetilde{G}, B)$.

At last, the following theorem is a basic property about soft fuzzy incline.

Theorem 3.8 If $(\widetilde{F}, A)$ and $(\widetilde{G}, B)$ are two soft fuzzy incline over the incline $\left(E,+,^{*}\right)$, then $(\widetilde{F}, A) \sim(\widetilde{G}, B)$ is also a soft fuzzy incline. 
Proof. Since $(\widetilde{F}, A)$ and $(\widetilde{G}, B)$ are soft fuzzy incline over the incline $\left(E,+,{ }^{*}\right)$, by Theorem 3.6, $\operatorname{EXT}_{A \cup B}(\widetilde{F}, A)$ and $\operatorname{EXT}_{A \cup B}(\widetilde{G}, B)$ are both soft fuzzy incline. Thus, for each $e \in A \cup B, \widetilde{F}_{A \cup B}^{*}(e)$ and $\widetilde{G}_{A \cup B}^{*}(e)$ are fuzzy subincline of $\left(E,+,^{*}\right)$. Therefore, for all $x, y \in E$ by Theorem 3.3, $\widetilde{F}_{A \cup B}^{*}(e)(x) \wedge \widetilde{F}_{A \cup B}^{*}(e)(y)$

$$
\leq \widetilde{F}_{A \cup B}^{*}(e)(x+y) \wedge \widetilde{F}_{A \cup B}^{*}(e)\left(x^{*} y\right)
$$

and $\widetilde{G}_{A \cup B}^{*}(e)(x) \wedge \widetilde{G}_{A \cup B}^{*}(e)(y)$

$$
\leq \widetilde{G}_{A \cup B}^{*}(e)(x+y) \wedge \widetilde{G}_{A \cup B}^{*}(e)\left(x^{*} y\right) .
$$

Suppose $\quad(\widetilde{F}, A) \tilde{\wedge}(\widetilde{G}, B)=(\widetilde{H}, A \cup B) \quad$, where $\widetilde{H}(e)=\widetilde{F}_{A \cup B}^{*}(e) \wedge \widetilde{G}_{A \cup B}^{*}(e)$ for every $e \in A \cup B$. Then for all $x, y \in E$,

$$
\begin{aligned}
& \widetilde{L}(e)(x) \wedge \tilde{L}(e)(y) \\
& =\left[\widetilde{F}_{A \cup B}^{*}(e) \wedge \widetilde{G}_{A \cup B}^{*}(e)\right](x) \wedge\left[\widetilde{F}_{A \cup B}^{*}(e) \wedge \widetilde{G}_{A \cup B}^{*}(e)\right](y) \\
& =\left[\widetilde{F}_{A \cup B}^{*}(e)(x) \wedge \widetilde{F}_{A \cup B}^{*}(e)(y)\right] \\
& \wedge\left[\widetilde{G}_{A \cup B}^{*}(e)(x) \wedge \widetilde{G}_{A \cup B}^{*}(e)(y)\right] \\
& \leq\left[\widetilde{F}_{A \cup B}^{*}(e)(x+y) \wedge \widetilde{F}_{A \cup B}^{*}(e)\left(x^{*} y\right)\right] \\
& \wedge\left[\widetilde{G}_{A \cup B}^{*}(e)(x+y) \wedge \widetilde{G}_{A \cup B}^{*}(e)\left(x^{*} y\right)\right] \\
& =\left[\widetilde{F}_{A \cup B}^{*}(e)(x+y) \wedge \widetilde{G}_{A \cup B}^{*}(e)(x+y)\right] \\
& \wedge\left[\widetilde{F}_{A \cup B}^{*}(e)\left(x^{*} y\right) \wedge \widetilde{G}_{A \cup B}^{*}(e)\left(x^{*} y\right)\right] \\
& =\left[\widetilde{F}_{A \cup B}^{*}(e) \wedge \widetilde{G}_{A \cup B}^{*}(e)\right](x+y) \\
& \wedge\left[\widetilde{F}_{A \cup B}^{*}(e) \wedge \widetilde{G}_{A \cup B}^{*}(e)\right]\left(x^{*} y\right) \\
& =\widetilde{L}(x+y) \wedge \widetilde{L}\left(x^{*} y\right) \text {. }
\end{aligned}
$$

So, $(\widetilde{F}, A) \widetilde{\wedge}(\widetilde{G}, B)$ is a soft fuzzy incline over the incline $\left(E,+,{ }^{*}\right)$.

\section{Application}

In the section, an application of soft fuzzy inclines, the attractiveness of the houses, will be discussed.

Suppose that $U$ is the set of houses under consideration. $E$ is the set of parameters. There are six houses in the universe set $U$ and seven parameters in the set $E$ given by:
$U=\left\{h_{l}, h_{2}, h_{3}, h_{4}, h_{5}, h_{6}\right\}, E=\left\{e_{l}, e_{2}, e_{3}, e_{4}, e_{5}, e_{6}, e_{7}\right\}$, where $e_{1}$ stands for the parameter 'expensive', $e_{2}$ stands for the parameter 'beautiful', $e_{3}$ stands for the parameter 'wooden', $e_{4}$ stands for the parameter 'in good repair', $e_{5}$ stands for the parameter 'modern', $e_{6}$ stands for the parameter 'in good repair', $e_{7}$ stands for the parameter 'large space'.

$$
A=\left\{e_{l}, e_{2}, e_{3}, e_{4}, e_{5}\right\}, B=\left\{e_{1}, e_{3}, e_{4}, e_{6}, e_{7}\right\},
$$

Soft fuzzy inclines $(\widetilde{F}, A)$ and $(\widetilde{G}, B)$ describe the attractiveness of the houses which Mr. $\mathrm{X}$ and Mr. Y considered and are given by, respectively,

$\widetilde{F}\left(e_{1}\right)=0.4 / h_{1}+0.3 / h_{2}+0.6 / h_{3}+0.4 / h_{4}+0.8 / h_{5}+0.9 / h_{6}$, $\widetilde{F}\left(e_{2}\right)=0.6 / h_{1}+0.4 / h_{2}+0.8 / h_{3}+0.6 / h_{4}+0.7 / h_{5}+0.3 / h_{6}$, $\widetilde{F}\left(e_{3}\right)=0.9 / h_{1}+0.2 / h_{2}+0.4 / h_{3}+0.4 / h_{4}+0.7 / h_{5}+0.6 / h_{6}$, $\widetilde{F}\left(e_{4}\right)=0.2 / h_{1}+0.7 / h_{2}+0.4 / h_{3}+0.7 / h_{4}+0.6 / h_{5}+0.3 / h_{6}$, $\widetilde{F}\left(e_{5}\right)=0.1 / h_{1}+0.2 / h_{2}+0.7 / h_{3}+0.9 / h_{4}+0.3 / h_{5}+0.1 / h_{6}$. $\widetilde{G}\left(e_{1}\right)=0.7 / h_{1}+0.6 / h_{2}+0.7 / h_{3}+0.8 / h_{4}+0.7 / h_{5}+0.9 / h_{6}$, $\widetilde{G}\left(e_{3}\right)=0.4 / h_{1}+0.6 / h_{2}+0.9 / h_{3}+0.2 / h_{4}+0.4 / h_{5}+0.6 / h_{6}$, $\widetilde{G}\left(e_{4}\right)=0.2 / h_{1}+0.1 / h_{2}+0.2 / h_{3}+0.3 / h_{4}+0.6 / h_{5}+0.4 / h_{6}$, $\widetilde{G}\left(e_{6}\right)=0.2 / h_{1}+0.3 / h_{2}+0.8 / h_{3}+0.8 / h_{4}+0.3 / h_{5}+0.3 / h_{6}$, $\widetilde{G}\left(e_{7}\right)=0.8 / h_{1}+0.4 / h_{2}+0.6 / h_{3}+0.4 / h_{4}+0.8 / h_{5}+0.2 / h_{6}$.

The soft fuzzy inclines $(\widetilde{F}, A)$ and $(\widetilde{G}, B)$ over the universe set $U$ can be showed in the following tables:

\begin{tabular}{|c|c|c|c|c|c|}
\multicolumn{7}{|c|}{ TABLE I $(\widetilde{F}, A)$} \\
\hline$U$ & $e_{1}$ & $e_{2}$ & $e_{3}$ & $e_{4}$ & $e_{5}$ \\
\hline$h_{1}$ & 0.4 & 0.6 & 0.9 & 0.2 & 0.6 \\
\hline$h_{2}$ & 0.3 & 0.4 & 0.2 & 0.7 & 0.2 \\
\hline$h_{3}$ & 0.6 & 0.8 & 0.4 & 0.4 & 0.7 \\
\hline$h_{4}$ & 0.4 & 0.6 & 0.4 & 0.7 & 0.9 \\
\hline$h_{5}$ & 0.8 & 0.7 & 0.7 & 0.6 & 0.3 \\
\hline$h_{6}$ & 0.9 & 0.3 & 0.6 & 0.3 & 0.1 \\
\hline
\end{tabular}

TABLE II $(\widetilde{G}, B)$
\begin{tabular}{|c|c|c|c|c|c|}
\hline$U$ & $e_{1}$ & $e_{2}$ & $e_{3}$ & $e_{4}$ & $e_{5}$ \\
\hline$h_{1}$ & 0.7 & 0.4 & 0.2 & 0.2 & 0.8 \\
\hline$h_{2}$ & 0.6 & 0.6 & 0.1 & 0.3 & 0.4 \\
\hline$h_{3}$ & 0.7 & 0.9 & 0.2 & 0.8 & 0.6 \\
\hline$h_{4}$ & 0.8 & 0.2 & 0.3 & 0.8 & 0.4 \\
\hline$h_{5}$ & 0.7 & 0.4 & 0.6 & 0.3 & 0.8 \\
\hline$h_{6}$ & 0.9 & 0.6 & 0.4 & 0.3 & 0.2 \\
\hline
\end{tabular}


TABLE III $(\widetilde{F}, A) \widetilde{\wedge}(\widetilde{G}, B)$

\begin{tabular}{|c|c|c|c|c|c|c|c|}
\hline$U$ & $e_{1}$ & $e_{2}$ & $e_{3}$ & $e_{4}$ & $e_{5}$ & $e_{6}$ & $e_{7}$ \\
\hline$h_{1}$ & 0.4 & 0.5 & 0.4 & 0.2 & 0.5 & 0.2 & 0.5 \\
\hline$h_{2}$ & 0.3 & 0.4 & 0.2 & 0.1 & 0.2 & 0.3 & 0.4 \\
\hline$h_{3}$ & 0.6 & 0.5 & 0.4 & 0.2 & 0.5 & 0.5 & 0.5 \\
\hline$h_{4}$ & 0.4 & 0.5 & 0.2 & 0.3 & 0.5 & 0.5 & 0.4 \\
\hline$h_{5}$ & 0.7 & 0.5 & 0.4 & 0.6 & 0.3 & 0.3 & 0.5 \\
\hline$h_{6}$ & 0.9 & 0.3 & 0.6 & 0.3 & 0.1 & 0.3 & 0.2 \\
\hline
\end{tabular}

TABLE IV $(\widetilde{F}, A) \widetilde{\vee}(\widetilde{G}, B)$

\begin{tabular}{|c|c|c|c|c|c|c|c|}
\hline$U$ & $e_{1}$ & $e_{2}$ & $e_{3}$ & $e_{4}$ & $e_{5}$ & $e_{6}$ & $e_{7}$ \\
\hline$h_{1}$ & 0.7 & 0.6 & 0.9 & 0.2 & 0.6 & 0.5 & 0.8 \\
\hline$h_{2}$ & 0.6 & 0.5 & 0.6 & 0.7 & 0.5 & 0.5 & 0.5 \\
\hline$h_{3}$ & 0.7 & 0.8 & 0.9 & 0.4 & 0.7 & 0.8 & 0.6 \\
\hline$h_{4}$ & 0.8 & 0.6 & 0.4 & 0.7 & 0.9 & 0.8 & 0.5 \\
\hline$h_{5}$ & 0.9 & 0.4 & 0.7 & 0.6 & 0.5 & 0.5 & 0.8 \\
\hline$h_{6}$ & 0.9 & 0.5 & 0.6 & 0.4 & 0.5 & 0.5 & 0.5 \\
\hline
\end{tabular}

From TABLE I and TABLE II, we can find soft fuzzy inclines $(\widetilde{F}, A)$ and $(\widetilde{G}, B)$ describe the satisfaction for Mr. $\mathrm{X}$ and Mr. $\mathrm{Y}$ with the attractiveness of the houses which they are going to buy, respectively. And soft fuzzy inclines $(\widetilde{F}, A) \wedge(\widetilde{G}, B)$ and $(\widetilde{F}, A) \widetilde{\vee}(\widetilde{G}, B)$ been shown in TABLE III and TABLE IV describe their common interests in the attractiveness. $(\widetilde{F}, A) \tilde{\wedge}(\widetilde{G}, B)$ describes their pessimistic views and $(\widetilde{F}, A) \widetilde{\vee}(\widetilde{G}, B)$ describes their optimistic views. Here, if a value is 0.5 , it describes that the man disinterests in the attractiveness of the houses. If greater than 0.5 , it describes the man is satisfied with the attractiveness of the house and is dissatisfied if less than 0.5 .

\section{ACKNOWLEDGMENT}

This paper supported by scientific research project of Xi'an Peihua University "Game Playing Analysis in Pursue Differentiated Market” (Grant No. PHKT17001) and basic research fund of Xi'an University of Architecture and Technology (Grant No. JC1709).

\section{REFERENCES}

[1] L. A. Zadeh, “Fuzzy sets,” Information and Control, vol. 8, pp. 338-353, 1965.

[2] M. B. Gorzalzany, "A method of inference in approximate reasoning based on interval-valued fuzzy sets,” Fuzzy Sets and Systems, vol. 21, pp. 1-17, 1987.

[3] W. L. Gau and D. J. Buehrer, "Vague sets,” IEEE Trans. System Man Cybernet, vol. 23, pp. 610-614, 1993.

[4] Z. Pawlak, "Rough sets," International Journal of Information and Computer Sciences, vol. 11, pp. 341-356, 1982.

[5] D. Molodtsov, "Soft set theory - first results," Comput. Math. Applic., vol. 37, pp. 19-31, 1999.

[6] P. K. Maji, R. Biswas and A. R. Roy, "Soft set theory,” Comput. Math. Applic., vol. 45, pp. 555-562, 2003.

[7] A. R. Roy and P. K. Maji, "A fuzzy soft set theoretic approach to decision making problems,” J. Comput. Appl. Math., vol. 203, pp. 412 418, 2007. 